\title{
Photoluminescent nanocluster-based probes for bioimaging applications
}

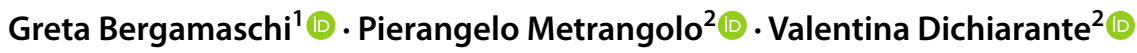

Received: 7 October 2021 / Accepted: 1 December 2021 / Published online: 15 January 2022

(c) The Author(s), under exclusive licence to European Photochemistry Association, European Society for Photobiology 2021

\begin{abstract}
In the continuous search for versatile and better performing probes for optical bioimaging and biosensing applications, many research efforts have focused on the design and optimization of photoluminescent metal nanoclusters. They consist of a metal core composed by a small number of atoms (diameter $<2-3 \mathrm{~nm}$ ), usually coated by a shell of stabilizing ligands of different nature, and are characterized by molecule-like quantization of electronic states, resulting in discrete and tunable optical transitions in the UV-Vis and NIR spectral regions. Recent advances in their size-selective synthesis and tailored surface functionalization have allowed the effective combination of nanoclusters and biologically relevant molecules into hybrid platforms, that hold a large potential for bioimaging purposes, as well as for the detection and tracking of specific markers of biological processes or diseases. Here, we will present an overview of the latest combined imaging or sensing nanocluster-based systems reported in the literature, classified according to the different families of coating ligands (namely, peptides, proteins, nucleic acids, and biocompatible polymers), highlighting for each of them the possible applications in the biomedical field.
\end{abstract}

\section{Graphical abstract}

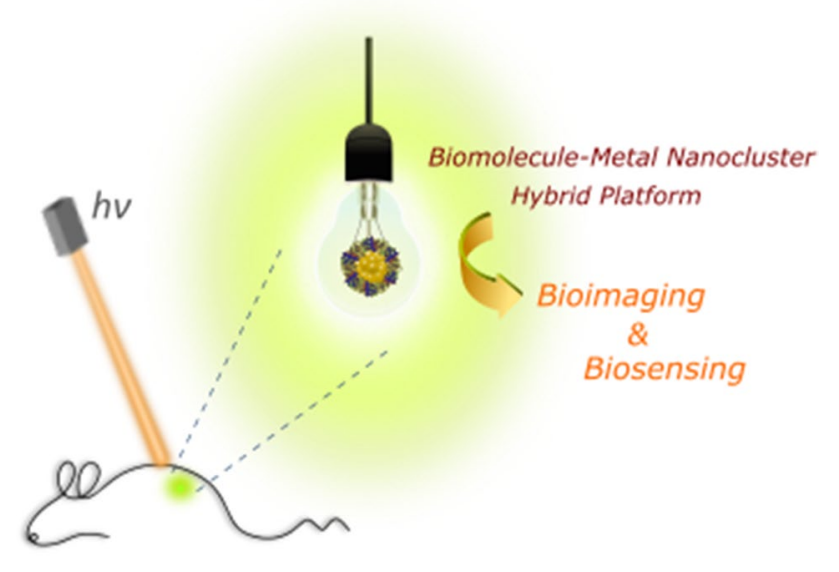

Keywords Metal nanoclusters $\cdot$ Optical bioimaging $\cdot$ Hybrid probes $\cdot$ Peptides $\cdot$ Proteins $\cdot$ Biopolymers

Dedicated to Prof. Angelo Albini on the occasion of his 75th birthday.

Valentina Dichiarante

valentina.dichiarante@polimi.it

Extended author information available on the last page of the article

\section{Introduction}

Optical bioimaging is currently recognized as a powerful and non-invasive method for obtaining biological information about the structures and dynamics of cells, tissues, and even whole living organisms, through the detection of 
different kinds of signals emitted by optically active probes. Among all the existing types of optical responses, fluorescence and luminescence are the most commonly exploited ones, both in vitro and in vivo [1]. Conventional fluorescent imaging probes usually consist of organic dyes, engineered fluorescent proteins, semiconductor quantum dots, or carbon dots [2-7]. Despite their good performance in terms of sensitivity, accuracy, and response time, the previously mentioned classes of tracers show severe limitations, concerning, for instance, their reduced photostability, difficult preparation, high cost, or potential toxicity [8]. For these reasons, the last years have seen a constantly increasing interest about the development of stable colloidal nanoparticles (NPs) as alternative luminescent materials [9]. In particular, gold nanoparticles proved to be an extremely promising platform for bioimaging, thanks to their easy fabrication, chemical stability, excellent biocompatibility, and tunable optical properties [10].

The increasing amount of research efforts, focused on the engineering of metal NPs with tailored shapes and sizes, has additionally led to the development of a peculiar sub-family of nanomaterials, termed "nanoclusters" (NCs), that are characterized by a single- or multi-metal core with a diameter lower than $2-3 \mathrm{~nm}$. Such NCs generally consist of a small number, from less than ten up to a few hundred, of noble metal atoms, mostly gold ( $\mathrm{Au}$ ), silver $(\mathrm{Ag})$, or copper $(\mathrm{Cu})$. Instead of the localized surface plasmon resonance (LSPR) typical of larger metal
NPs, NCs show a molecule-like quantization of electronic states, which results in discrete optical transitions in the ultraviolet-visible (UV-Vis) and near infrared (NIR) spectral regions. Since naked metal NCs have a strong tendency to aggregate irreversibly, most of the times organic or inorganic protecting ligands are used to stabilize their surface [11]. Similarly to quantum dots, metal NCs show quite intense fluorescence bands, with broad stokes shifts, and with emission wavelengths that can be tuned from UV-Vis to NIR simply by changing the number and the nature of both core atoms and stabilizing ligands. Noteworthy, in addition to their interesting optical behavior, NCs exhibit low toxicity, due to the fact that their ultrafine sizes seem to favor a fast removal from the body and to avoid their uptake by the reticuloendothelial system [12]. All these features appear extremely attractive in view of the application of metal NCs in biological imaging and fluorescence labeling, thanks also to recent advances in the size-selective synthesis of tailored cluster cores, and in their surface functionalization or conjugation with biologically relevant tags or targeting moieties [13, 14].

After an initial brief description of the key structural and optical properties of metal NCs, this Review will focus mainly on hybrid imaging probes obtained by the combination of NCs with different classes of biomolecules (e.g., peptides, proteins, nucleic acids, etc.) and will present an overview of the most relevant and recently reported examples in the field (Fig. 1).

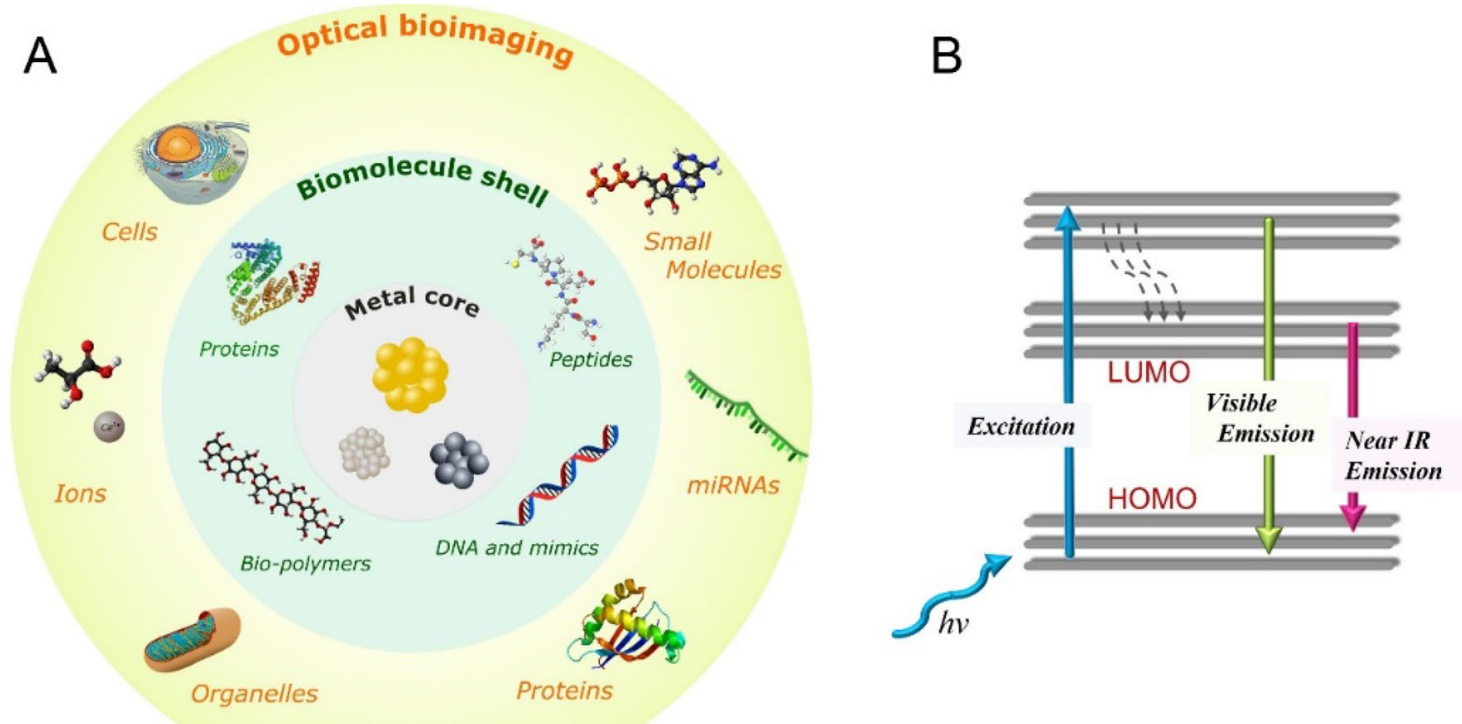

Fig. 1 Schematic illustration of biomolecule-templated metal NCs and the related optical bioimaging and biosensing applications (a). Schematic diagram of metal NCs discrete orbitals, showing the possible excitation and relaxation pathways (b) 


\section{Metal nanoclusters}

\subsection{Structural features and synthetic strategies}

Thanks to the ultra-small diameter of their metal core, ranging from the sub-nanometer scale to a maximum of 2-3 nm, nanoclusters can actually be considered intermediate species between discrete organic molecules, or organometallic compounds, and plasmonic NPs. The latest synthetic improvements have made possible to prepare several metal NCs with atomically precise structure, and a chemical composition described by a definite formula, instead of an average size distribution typical of bigger NPs. Size selection seems to be favored by the presence of preferential thermodynamic paths, that lead to the socalled "magic number" clusters (e.g., $\mathrm{Au}_{22}, \mathrm{Au}_{25}, \mathrm{Ag}_{14}$ ), whose metallic cores exhibit high stability due to the closing of their outer electronic shells [15].

The synthesis of metal NCs can be achieved by different routes. The direct reduction of metal salts (e.g., $\mathrm{Au}$ (III) or $\mathrm{Ag}(\mathrm{I}))$ by sodium borohydride, in the presence of thiols, has been successfully optimized for the synthesis of $\mathrm{Au}$, $\mathrm{Ag}, \mathrm{Cu}$, and alloy NCs in both organic and aqueous media. Chemical etching of pre-synthesized larger NPs or NCs by removal of metal atoms from the outermost layer was shown to form more homogeneous smaller NCs. Ligand exchange of a pre-synthesized NCs, as well, proved its effectiveness in modulating the structure and properties of $\mathrm{NCs}$ in terms of geometry, bonding, and electronic transitions [16]. Finally, the so-called "template method"- that consists in the reduction of metal salts by a polymer or a macromolecule able to bind metal ions-has become of primary importance for the preparation of NCs. Several biomolecules-e.g., nucleic acids, amino acids, proteins, peptides, and enzymes-have been used as templates, and provided NCs not only with improved biocompatibility and water solubility but also with optical features more suitable for bioimaging applications, such as large Stokes shifts and NIR-centered emission bands. Template molecules may also be useful for allowing further surface functionalization of NCs through conjugation to other molecules and biomolecules $[9,11,16]$.

Core and surface are quite entangled entities in the structure of NCs, with some metal atoms actually shared between the core and the outer shell. This makes very challenging to study them separately, mapping their structure-property correlations and understanding their specific functional roles. Since any change in the core structure would inevitably affect also the NC surface, and vice versa, it is of fundamental importance to develop tailored approaches for the selective structural modification of the core or the surface in NCs. Similarly to plasmonic
NPs, gold has generally been the preferred candidate as core metal also for NCs, thanks to its high stability toward oxidation. It is not thus surprising that the goal of synthesizing monodisperse NCs with atomic-level precision has been achieved for the first time using this noble metal [10]. Despite a lower redox stability, indeed, other metals, like $\mathrm{Ag}$ and $\mathrm{Cu}$, take advantage of their higher abundance and reduced cost. For this reason, doping of the Au core with the introduction of one or more different metal atoms (e.g., $\mathrm{Pd}, \mathrm{Pt}, \mathrm{Ag}$ ) has also been pursued as a strategy to improve and tune the optical properties of the resulting alloy NCs, by varying the number of dopant atoms and the distribution of doping sites in the cluster core [17].

\subsection{Optical properties}

The photophysical properties of metal nanoclusters are extremely peculiar and attractive from several viewpoints. In particular, their ability to emit photoluminescence (PL) in the IR, or even NIR, field is a feature not so common either in their molecular counterparts or in larger NPs. This emission might be exploited not only as a probe of the electronic behavior at the nanoscale but also for biological imaging and chemical sensing. The very small size of NCs, close to the Fermi wavelength of electrons in metals, is known to induce a drastic reduction of the collective oscillation of free electrons in the metal core, with the consequent loss of LSPR effect. The continuous band structure of atoms in bulk metals becomes split into discrete orbitals, where free electrons are confined, in the case of NCs. This quantum size effect is responsible of their molecule-like behavior, characterized by discrete HOMO-LUMO gaps, step-like absorption bands, quite intense PL, unusual redox properties, and in some cases even chirality and intrinsic magnetism [18].

Although the exact origin of the photoluminescence of metal NCs is still under debate, and this has somehow limited their practical applications, two major mechanisms seem to contribute to this phenomenon. The first one is the metal quantum confinement effect described above, according to which the emission of NCs should be due to intra-band (sp-sp) and inter-band (sp-d) transitions among their discrete energy levels. The second key factor is a surface charge transfer deriving from the interaction between stabilizing ligands and the metal core, which could consist in ligand-tometal charge transfer (LMCT) or ligand-to-metal-to-metal charge transfer (LMMCT) [19]. Initially, in fact, the PL of Au NCs was found to shift from UV to NIR for different cluster sizes, suggesting the hypothesis that it was originated from the metal core only. According to these observations, a simple Jellium model was proposed to describe the size dependence of the emission maxima of Au NCs, as described by Eq. (1): 
$h v \cong \frac{E_{\mathrm{F}}}{\sqrt[3]{N}}$

where $E_{\mathrm{F}}$ is the Fermi energy of bulk gold and $N$ is the number of Au atoms in the cluster [20].

Interestingly, more recent literature reports seem to indicate that emission quantum yields, energies, and lifetimes in NCs are quite independent from their diameter, whereas they depend heavily on their surface chemistry, including both the metallic composition and the ligand shell's architecture. Different shell geometries, for example, may influence the polarization of the metal-ligand interface, as well as facilitate ligand-to-metal charge transfer processes, and both factors have been demonstrated to influence emission properties. Similarly, the formation of alloy NCs proved to be a powerful tool for altering their surface metal composition, and consequently for tuning their PL [21]. To date, the most likely explanation of PL mechanism in NCs seems to require a compromise model, which takes into account all the different contributions arising from metal-ligand interface chemistry. Core size and types of protecting ligands, as well as valence electron count, oxidation state of the metal, crystal structure, temperature, and $\mathrm{pH}$, appear all crucial factors in controlling the PL of NCs [19].

Unfortunately, although emission quantum yields (QYs) of metal NCs are higher if compared to NPs or bulk metals, PL of NCs-especially of thiol-stabilized ones-is generally weak, with QYs rarely exceeding $0.1 \%$. Furthermore, the high surface-to-volume ratio typical of these ultra-small particles often leads to an easy quenching of their photoluminescence in solution by solvent molecules or oxygen. For this reason, many NCs do not emit at room temperature, but only at low temperatures, or even in gas matrix as in the case of bare Au and Ag NCs. Such issue has deeply limited their practical application in the biomedical field thus far. Aggregation-induced emission (AIE) has been lately proposed as an efficient strategy for improving NCs' photoluminescence performance by directing their assembly [22]. Contrary to organic dyes, whose aggregation usually quenches PL due to the formation of excimers, the emission intensity of AIE-active molecules (AIE-gens) is strongly enhanced in the solid or aggregated state, thanks to restricted intramolecular vibrations and rotations. The implementation of AIE phenomenon in the NC field might thus allow the design of highly luminescent metal nanoclusters, as already reported for some thiolated $\mathrm{Au}$ and $\mathrm{Ag} \mathrm{NCs}$ whose QYs increased up to 5-20\% [22]. In addition to usual single-photon absorption, metal NCs can be excited by two photons, whose energy is a half compared to single-photon excitation and generally corresponds to absorption in the NIR region. The resulting high penetration depth and limited light loss in living samples would be extremely beneficial for low power medical imaging, since the provided anti-Stokes emission enhances the signal-to-noise ratio, avoiding the excitation of organic fluorophores present in biological matrixes. Moreover, two-photon excited fluorescence could also be effective in avoiding photobleaching and phototoxicity phenomena typical of single-photon fluorescence [23]. Recent studies performed on gold NCs with different core sizes confirmed their large two-photon absorption cross section, making them promising tools for multi-photon bioimaging. In particular, thiolated Au NCs showed cross sections in the range of $10^{5}-10^{6} \mathrm{GM}$ units $\left(1 \mathrm{GM}=10^{-50} \mathrm{~cm}^{4} \mathrm{~s}_{\text {photon }}{ }^{-1}\right)$ upon excitation at $800 \mathrm{~nm}$, which are extremely higher if compared to the $\sim 1000 \mathrm{GM}$ values typical of two-photon organic macromolecular chromophores [24-27]. Finally, it is worth to notice that the creation of optical quality films of NCs could increase the interactions among them, and result in further enhancement of both their linear and non-linear (NLO) optical responses [28, 29].

\subsection{Applications}

The unique electronic and optical properties of metal NCs are clearly related to the promising potential of these nanomaterials for photo- and electro-catalytic applications, as recently reviewed in detail by Xie et al. [30]. The latest successful achievements in the preparation of atomically precise NCs have opened up exciting opportunities for fundamental catalysis, since the possibility to have well-defined catalytic species could allow a molecular-level understanding of catalytic mechanisms at the nanoscale. Furthermore, a broad range of ligand-protected metal NCs and solid-supported metal clusters with high catalytic activity could be engineered for different aims, from enantioselective heterogeneous catalytic processes to photocatalysis [31]. Atomically precise metal nanoclusters exhibit peculiar electrochemical properties, with redox potentials that are very sensitive to their composition, shape, and size. They could thus be exploited as efficient electron transfer mediators for targeted electrocatalysis, for example, or represent a valuable alternative to low band-gap semiconductors, avoiding issues related to photochemical or electrochemical corrosion [32]. NCs have been also studied as potential nanoscale building blocks to create three-dimensional superstructures through colloidal self-assembly, thanks to their spontaneous organization into highly ordered functional nanostructures. These self-assembled architectures might be exploited for harvesting, and thus enhancing, the collective optical properties of all neighboring NCs, as well as for creating confined nanocavities for drug delivery, directed synthesis, or selective catalysis [33, 34]. 
One of the most widely studied applications of luminescent NCs composed of $\mathrm{Au}, \mathrm{Ag}, \mathrm{Cu}$, and $\mathrm{Pt}$ is their use as chemosensors for the detection of several specific analytes, e.g., metal ions, anions, biomolecules, proteins, and nucleic acids, as well as $\mathrm{pH}$-sensing probes or optical sensors for the detection of reactive oxygen species (ROS) [35]. The biosensing ability of metal NCs have raised interest toward their possible role in the biomedical field as promising theranostic agents, thanks to their favorable physicochemical properties. In particular, the ultra-small size of NCs can facilitate tumor targeting and renal clearance, and their peculiar luminescence looks promising for constructing bioimaging probes, and even traceable controlled-release drug-delivery systems for imaging-guided therapy. Excitation of the metal core of $\mathrm{NCs}$ upon absorption of ionizing radiation (e.g., accelerated electrons, gamma rays, $\mathrm{X}$-rays, etc.), for example, emits secondary electrons useful for radiotherapy (RT), whereas light absorption can generate ROS, required for photodynamic therapy (PDT) [36]. Fluorescence and phosphorescence, that can coexist in the same NC species, normally have lifetimes in the range of $0.1-10 \mu \mathrm{s}$, that are orders of magnitude longer than the self-fluorescence of biological systems (usually $<10 \mathrm{~ns}$ ). Moreover, NCs could be suitable for in vivo bioimaging, since they can emit in the NIR wavelength range, where the reduced optical scattering in biological tissues allows deep penetration of photons, and increased resolution. Finally, a proper tailoring of surface coverage would permit to tune the interactions of NC-based probes with cells, reaching an optimal balance between their membrane binding tendency and cellular uptake [37].

\section{Hybrid nanocluster-based imaging probes}

As previously mentioned, strongly coordinating ligandse.g., sulfhydryl compounds and polymers-have been used in the synthesis of metal NCs to control their size, and to inhibit their agglomeration during the growth step. Despite the relatively good stability of NCs prepared by these methods, their biocompatibility still needs to be improved for biomedical purposes. If, on one side, both in vitro and in vivo studies indicate that exposure to larger metal NPs could lead to their accumulation in some organs-e.g., heart, liver, spleen, kidney, and brain — after inhalation, ingestion or skin contact, as well as induce production of ROS, much less is currently known about the toxicity of NCs [38, 39]. Recent literature reports on gold nanoclusters, especially those conjugated to proteins, seem to agree on a reduced impact in vivo, thanks to their smaller size and subsequent faster renal clearance [40-42].

Biomolecules, such as DNA, proteins, and peptides, as well as other biocompatible polymers, contain several functional groups with a strong affinity for metal atoms, and can thus act simultaneously as stabilizing and reducing agents, that convert metal ions to metal atoms. In addition to better biocompatibility, biomolecule-protected NCs allow a wider selection of ligands, whose structure and functions are generally preserved after the synthesis $[43,44]$. Besides the advantages afforded by their use as templating agents, biomolecules play a prominent role also in endowing metal NCs with specific recognition capability for certain biomarkers, generating versatile multifunctional hybrid platforms for bioimaging applications [45]. Actually, the design of stable bioimaging probes with real-time monitoring ability, effective targeting, and good biocompatibility is highly desirable and could significantly contribute to improve early diagnosis and treatment of diseases. In particular, the functionalization of metal NCs with specific biomolecules, combined with their inherent fluorescence, may result in a synergistic enhancement of their subcellular localization and imaging performance [46-48].

This section will present an overview of the most recent significant examples of hybrid metal nanocluster-based systems, classified according to the different biotemplates that they contain, as summarized in Table 1. We will focus on the use of such platforms as smart tools for bioimaging assays, with tunable luminescence properties that can allow the detection of specific biomarkers or the monitoring of physiological properties, both in vitro and in vivo.

\subsection{Peptides}

Peptides are short chains of amino acids (usually no more than 50 residues) connected by amide bonds, which are naturally present in various organisms, where they exert specific biological functions. Differently from larger proteins, rationally designed peptides with specific amino acid sequences, and consequently with specific functionalities, can be easily obtained through solid-phase synthetic techniques [49]. Over the past few decades, peptides have been widely used as stabilizing ligands in the synthesis of metal NCs, thanks to their simple composition and well-organized structures [50, 51]. Peptides containing several reactive sequences or amino acids, indeed, can provide NCs with different simultaneous functional properties, such as selective targeting abilities, or $\mathrm{pH}$ responsiveness [52]. For these reasons, the conjugation of peptides to metal NCs represents a viable strategy to create hybrid imaging platforms with improved selectivity, biocompatibility, and target binding ability, which are all highly required skills for biomedical applications [53, 54].

Among all the available functional moieties provided by amino acids, peptides containing thiolate groups have been successfully used to prepare a variety of metal NCs, thanks to the formation of strong metal-S bonds involving their cysteine (Cys) residues (metal $=\mathrm{Au}, \mathrm{Ag}, \mathrm{Cu}$, etc.). One of the most widely exploited examples in this sense is 
Table 1 List of hybrid NC probes cited in the manuscript and their applications

\begin{tabular}{|c|c|c|c|}
\hline Ligand biomolecules & Core metal & Applications & Refs \\
\hline \multicolumn{4}{|l|}{ Peptides } \\
\hline GHS & $\mathrm{Cu}$ & Imaging nuclear membranes, detection of $\mathrm{Fe}^{3+}$ ions & [56] \\
\hline GHS & $\mathrm{Au}$ & Temperature sensing and cellular imaging & [57] \\
\hline mercaptan acids & $\mathrm{Au}$ & Amyloid detection in living cell & [58] \\
\hline poly-Arg & $\mathrm{Au}$ & Enzyme quantification in biological fluids, cancerous cell imaging & [59]-[60] \\
\hline $\mathrm{CCY}$ & $\mathrm{Ag} / \mathrm{Au}$ & Quantification of $\mathrm{ClO}^{-}$in living cells & [63] \\
\hline SV & $\mathrm{Au}$ & Multimodal cell imaging & {$[64]$} \\
\hline CCY-SAP & $\mathrm{Au}$ & Labeling of integrin-rich cells & {$[65]$} \\
\hline cyclic RGD & $\mathrm{Au}$ & Targeting of tumor cells in vitro and in vivo & {$[66]$} \\
\hline \multicolumn{4}{|l|}{ Proteins } \\
\hline BSA & $\mathrm{Au}$ & Detection of ROS in liver cells & [72] \\
\hline FITC-BSA & $\mathrm{Au}$ & Temperature and $\mathrm{pH}$ probing & [73] \\
\hline FA-BSA & $\mathrm{Au}$ & Active targeting of ovarian cancer cells & [75] \\
\hline CTPR & $\mathrm{Au} / \mathrm{Cu} / \mathrm{Ag}$ & Live cell imaging and labeling & [76] \\
\hline CTPR390 & $\mathrm{Au}$ & Detection of fibrosis disease in vitro, and in vivo & [77] \\
\hline RNaseA & $\mathrm{Au}$ & Imaging of GI tract and tumors in vitro and in vivo & [78] \\
\hline \multicolumn{4}{|c|}{ DNA and related synthetic mimics } \\
\hline APT & $\mathrm{Ag}$ & Quantification of MUC1 in serum and imaging in breast cancer cells & [88] \\
\hline DNA & $\mathrm{Ag}$ & Detection of TBI-related miRNA & [91] \\
\hline Apt-DNA & $\mathrm{Au}$ & Monitoring TK1 mRNA expression in vitro and in vivo & [92] \\
\hline \multicolumn{4}{|c|}{ Biocompatible polymers } \\
\hline PEG-PNIPAm & $\mathrm{Au}$ & Imaging of vesicular structures & [93] \\
\hline PCIE & $\mathrm{Au}$ & Bioimaging in vitro and in vivo & [95] \\
\hline $\mathrm{NAC}-\mathrm{CS}$ & $\mathrm{Au}$ & Bioimaging in vitro and in vivo & [96] \\
\hline $\mathrm{ACD}$ & $\mathrm{Pt} / \mathrm{Ag}$ & Detection of tumor cells in vitro and in vivo & [97] \\
\hline GNPs & $\mathrm{Au}$ & Biological tissue imaging & [98] \\
\hline $\mathrm{p}(\mathrm{NAm})$ & $\mathrm{Au}$ & Selective detection MDA-MB-231 cells & [99] \\
\hline $\mathrm{CNCs}$ & $\mathrm{Au}$ & Optical fibers for biological tissues & {$[100]$} \\
\hline
\end{tabular}

glutathione (GSH), a tripeptide that plays a crucial role as templating and stabilizing agent for metal NCs [55]. Das et al. introduced an efficient protocol for the one-pot synthesis of blue-emitting luminescent $\mathrm{Cu}$ NCs, using GSH as protecting group and reducing agent at the same time. Differently from previously reported methods, the reducing capability of the - $\mathrm{SH}$ group in glutathione was strongly enhanced by using alkaline reaction conditions $(\mathrm{pH}=10)$. The resulting GSH-Cu NCs exhibited stable luminescent properties at different $\mathrm{pH}$ values, good biocompatibility toward cell growth, and high photostability. Cell imaging studies revealed that GSH-Cu NCs mostly localized in the nuclear membranes of different tumor cells, making them a potential novel probe for understanding the dynamics involved in cancer cell division. Besides this, GSH-Cu NCs could also be used as metal ion sensors, since they were able to detect $\mathrm{Fe}^{3+}$ ions in solution even at nanomolar concentrations [56]. Furthermore, Zhang et al. found that glutathione-capped gold nanoclusters (GSH-Au NCs) possessed a thermo-responsive behavior, and could be employed as potential nanothermometers in living cells, with a considerable temperature resolution $\left(0.73^{\circ} \mathrm{C}\right)$ in all the range of physiological temperatures $\left(35-42{ }^{\circ} \mathrm{C}\right)$ [57].

The ligand exchange methodology allowed to obtain various highly fluorescent $\mathrm{Au}$ NCs coated by mercaptocarboxylic acids with different carbon chain lengths. By using 3-mercaptopropionic acid, 6-mercaptohexanoic acid, 11-mercaptoundecanoic acid, and 16-mercaptohexadecanoic acid as template agents, for instance, it was possible to achieve a fine modulation of the NC surface hydrophobicity, as well as to tune their emission maxima from 510 to $650 \mathrm{~nm}$ (Fig. 2). Cell viability and uptake experiments performed on fibroblast cell lines proved the high biocompatibility of such gold nanoclusters. Interestingly, these new platforms were also efficiently tested for monitoring protein aggregation phenomena associated with amyloidosis, without the use of any additional fluorescent marker (e.g., thioflavin) [58].

The conjugation of GSH-Au NCs with poly-arginine (poly-Arg) fragments have been applied to develop new AIE-labeling systems for targeting analytes in biological 


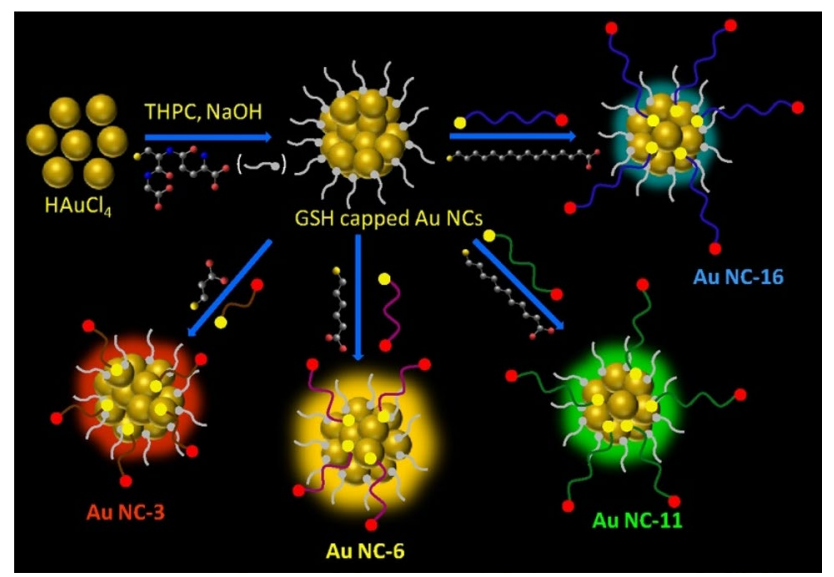

Fig. 2 Pictorial scheme of the ligand exchange synthesis of Au NCs functionalized with different mercaptocarboxylic acids. Orange-, yellow-, green-, and cyan-emitting nanoclusters have been obtained by using 3-mercaptopropionic acid, 6-mercaptohexanoic acid, 11-mercaptoundecanoic acid, and 16-mercaptohexadecanoic acid, respectively, as templating agents. Reproduced with permission from ref. [58]

fluids. Using four different arginine-based peptidesnamely, AG73, GR, pYR, and RGDR5-it was possible to obtain probes with different selectivity. Interestingly, these biosensors allowed quantitative determination of heparin (AG73-GSH-Au NCs), human trypsin (GR-GSH-Au NCs), and human alkaline phosphatase (pYR-GSH-Au NCs) in plasma and urine, as well as the imaging of $\alpha v \beta 3$ integrinoverexpressing cancer cells (RGDR5-GSH-Au NC). Moreover, compared to GSH-protected Au NCs, poly-R conjugated ones exhibited higher quantum yields and prolonged luminescence lifetimes [59]. The potential effectiveness of cationic arginine-stabilized Au NCs (AuSG-2Arg) as diagnostic probes was investigated in cancerous cells lines by Broekgaarden et al. The study confirmed that, despite a limited radiotherapeutic potential, AuSG-2Arg were more efficient diagnostic agents, compared to GSH-Au analogs, thanks to their enhanced uptake in glioblastoma models and increased emission intensity at $670 \mathrm{~nm}$ [60].

The CCY (Cys-Cys-Tyr) domain has proven to be another typical sequence that promotes the synthesis of metal NCs, thanks to the ability of tyrosin's hydroxyl group to reduce metal ions, and the following stabilization effect of the formed cluster exerted by the cysteine' SH moiety $[61,62]$. To improve the cell penetrating ability of silver/ gold bimetallic nanoclusters, Jia and colleagues designed a CCY- $\gamma$-ECGRGRKKRRQRRR peptide, composed of domains having different roles. While the CCY moiety promoted the formation of $\mathrm{Ag} / \mathrm{Au}$ alloy NCs, the RGRKKRRQRRR sequence acted as a cell-penetrating probe through its lysosome-targeting sequence GRKKRRQRRR, and the $\gamma$-ECG fragment was able to interact with ROS, a

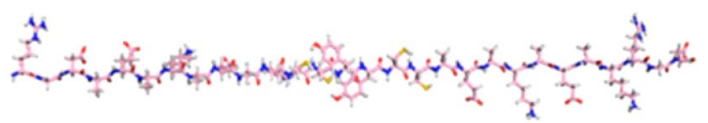

RGDAEAKAEAKCCYYCCAEAKAEAKRGD

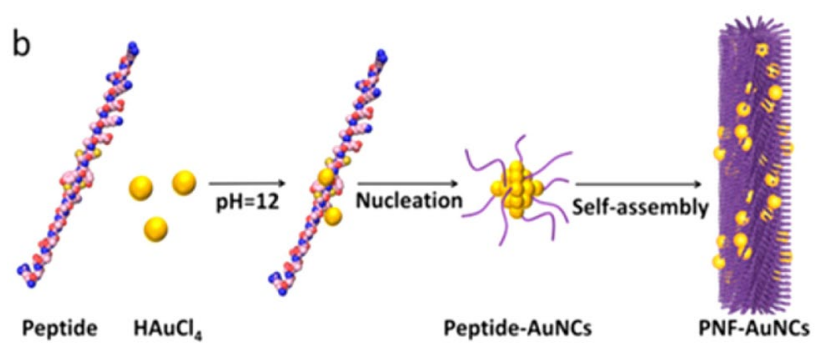

Fig. 3 Structure and amino acid sequence of a self-assembling peptide template (a), and key steps of the spontaneous formation of Peptide Nanofiber-Au NCs (PNF-Au NC) (b). Reproduced with permission from ref. [65] (further permissions related to the material excerpted should be directed to the American Chemical Society)

improving the stability of NCs in biological environments. Moreover, these fluorescent probes could also be useful for the detection of hypochlorite anion $\left(\mathrm{ClO}^{-}\right)$in living cells, whose presence resulted in fluorescence quenching, redshifted emission maximum, and decreased lifetime [63]. By changing the domain sequence of the capping peptides, it was possible to modulate the binding specificity of metal NCs toward a precise biological target, or cellular organelle. For example, the choice of a nuclear localization

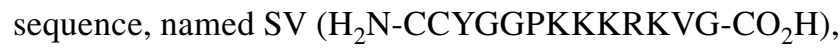
permitted to synthesize Au NCs which were easily transported into the cell nucleus. Optimizing synthetic conditions (e.g., light, $\mathrm{pH}, \mathrm{Au} /$ peptide ratio, reaction time and temperature), Liu et al. were able to fine-tune the emission properties of SV-capped gold nanoclusters, obtaining two distinct $\mathrm{Au} \mathrm{NC}$ families, characterized by blue $\left(\lambda_{\mathrm{ex}}=320 \mathrm{~nm}, \lambda_{\mathrm{em}}=405 \mathrm{~nm}\right)$ and red fluorescence emission $\left(\lambda_{\mathrm{ex}}=560 \mathrm{~nm}, \lambda_{\mathrm{em}}=657 \mathrm{~nm}\right)$, respectively. Such SV-Au NCs exhibited an excellent multimodal imaging ability, enabling dual-color imaging even by naked eye, and moreover, they showed effective peroxidase-like activity in the oxidation of tetramethylbenzidine [64]. The association between CCY domains with self-assembling peptide motifs afforded supramolecular structures of $\mathrm{Au}$ NCs with improved luminescence. For example, peptide sequence RGDAEAKAEAKCCYYCCAEAKAEAKRGD was successfully employed as multifunctional template for generating Peptide Nanofiber - Au NCs (PNF-Au NCs) superstructures. The AEAKAEAK moiety, containing both hydrophobic and hydrophilic residues, induced the spontaneous assembly of water-soluble fibril architectures (Fig. 3), characterized by a remarkable AIE effect that promoted a 70-fold enhancement in luminescence compared to unassembled Au NCs. Moreover, thanks to the 
presence of the RGD fragment, PNF-Au NCs were easily internalized by integrin-rich tumor cells via simple endocytosis [65].

Multimodal imaging of both extracellular and intracellular cancer biomarkers is a smart approach for cancer diagnosis and therapy. To design a suitable multimodal targeting probe, Chen et al. double conjugated Au NCs with cyclic RGD (cRGD), that enabled the detection of tumor tissue, and with aptamer AS1411 (Apt), that improved the affinity to nucleolin, another protein which is overexpressed in the cytoplasmic region of tumor cells. Au NC-cRGD-Apt has been further functionalized with a NIR-emitting derivative of indocyanine green $\left(\lambda_{\mathrm{em}} \sim 810 \mathrm{~nm}\right)$. The resulting NIR fluorescent dual-targeting probe displayed low cytotoxicity, and favorable tumor-targeting capability both in vitro and in vivo, suggesting its potential use for clinical cancer imaging [66].

\subsection{Proteins}

Proteins are biomolecules with a highly organized and complex three-dimensional structure, that play many critical roles in living organisms, such as providing structural integrity and taking part in the most relevant biochemical processes. The integration of the advantageous features of metal NCs and the active functional groups of proteins within a single system looks extremely useful for the development of tracking agents. A wide range of proteins have been employed in the synthesis of NC-based bioimaging probes, including hemoglobin, human serum albumin, lactoferrin, and resilin-mimetic proteins [67-70].

One of the first examples of protein-NC platform was reported by Xie et al., and exploited the ability of $\mathrm{Y}$ and $\mathrm{C}$ residues in bovine serum albumin (BSA) to reduce and stabilize gold ions, to obtain highly fluorescent gold nanoclusters [71]. As this synthetic approach is feasible, simple, and sustainable, BSA has been widely used as a template to synthesize NCs for biomedical applications. Jain et al. recently investigated the suitability of BSA-Au NCs as imaging nanoprobes for human hepatic cell line (WRL-68), observing that the designed Au nanoclusters were highly biocompatible and predominantly localized in the cytoplasm of WRL-68 cells. Moreover, the bright red fluorescence of absorbed BSA-Au NCs was sensitive to the exposure to a specific inhibitor of catalase enzyme, named 3-AT, involved in the generation of free radical species in cells. Thus, BSAAu NCs could be effectively used for the detection of hydrogen peroxide levels in the cytoplasm [72]. In another study, the integration of $\mathrm{pH}$-sensitive fluorescent dye fluorescein5-isothiocyanate (FITC) into BSA-Au NCs afforded a biocompatible temperature- and $\mathrm{pH}$-responsive fluorescent probe. FITC-BSA-Au NCs showed low cytotoxicity, good cell permeability, and two emission peaks - at $525 \mathrm{~nm}$ and
$670 \mathrm{~nm}$, respectively - that could be helpful for monitoring the simultaneous variations in temperature and $\mathrm{pH}$ in $\mathrm{HeLa}$ cells [73].

An effective strategy to image tumor cells is to directly target certain antigens or receptors that are overexpressed on their surface. As a result, this kind of approach can lead to an increase in probe delivery efficiency, and improved imaging detection quality both in vitro and in vivo. Folic acid (FA), for example, is a well-known selective ligand for the folate receptor $\alpha(\alpha-\mathrm{FR})$, which is overexpressed in various cancer cells, but barely found in healthy tissues [74]. In order to improve the active targeting of human ovarian adenocarcinoma cells (NIH:OVCAR-3) by Au NCs, BSA was functionalized with folic acid by chemical conjugation, obtaining FA-BSA-Au NC fluorescent probes with improved cellular uptake and staining ability compared to BSA-Au NCs. Intracellular localization was monitored by confocal fluorescence lifetime imaging microscopy [75].

Small engineered protein domains (usually comprising $\leq 100$ residues) show great promise as potential nextgeneration bioimaging tools, because their sequence and structure can be modified to improve targeting performance or intracellular localization. Within this category, it is worth to cite the tetratricopeptide repeating proteins (TPR or CTPR), characterized by repeating units of 34 amino acids, which form two antiparallel helices joined by a turn region. CTPR proteins can be engineered to display new functionalities and specific binding sites on the alpha-helix or in the loop portion. By incorporation of proper metal coordination sites, Aires et al. developed a new CTPR protein that enabled the synthesis of $\mathrm{Au}, \mathrm{Ag}$, and $\mathrm{Cu}$ NCs. The introduction of two His groups at positions 5 and 9 of CTPR $\alpha$-helices (Fig. 4) created a high-affinity bidentate motif for transition metal ions. The resulting set of different protein-stabilized NCs (Prot-NCs) displayed high photoluminescence, photostability under physiological conditions, and good biocompatibility. Moreover, Prot-NCs were internalized into breast cancer cells without any preliminary permeabilization treatment, and allowed efficient live cell imaging and labeling without any damage to cells or quenching phenomena [76]. The same Authors succeeded in developing a further multifunctional chimeric protein, called 4-AuNC, by fusion of fluorescent Prot-Au NCs with a protein recognition module (CTPR390) for in vivo imaging of chaperon Hsp90, which is involved in cardiovascular damages, such as hypertrophy and fibrosis diseases [77].

In the context of achieving non-invasive technologies for early in vivo diagnosis of pathological conditions, fluorescent probes emitting in the second near infrared window (NIR-II, 1000-1400 nm) have attracted substantial research enthusiasm, due to the fact that they would allow deep tissue penetration, as well as strongly reduced self-fluorescence and photon scattering. Gold nanoclusters encapsulated in the 


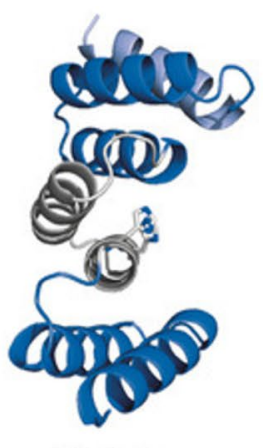

Protein

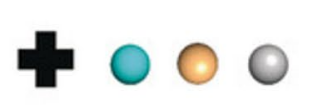

$\mathrm{Cu}, \mathrm{Au}$, or $\mathrm{Ag}$

Metal salt

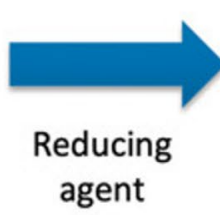

agent

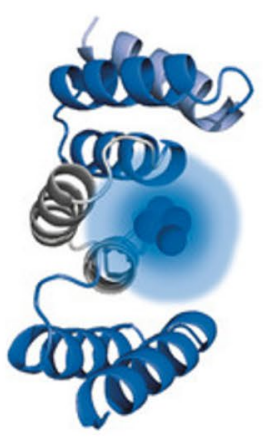

Protein-NC

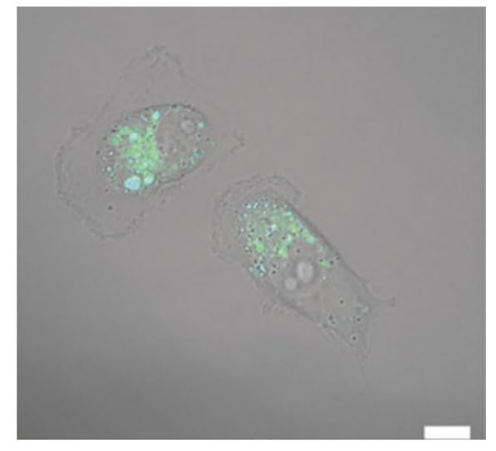

Fig. 4 Schematic illustration of the synthesis of fluorescent metal NCs templated by CTPR proteins (left). Live confocal fluorescence microscopy images of MDA-MB-231 breast cancer cells incubated

ribonuclease-A (RNaseA-Au NCs) protein corona showed red-shifted emissions around $1050 \mathrm{~nm}$, with excellent biocompatibility and photostability when exposed to gastrointestinal tract (GI) fluids or mammalian cells. In particular, compared to other NIR-II emitters, RNaseA-Au NCs migrated homogeneously during gastrointestinal peristalsis, allowing a detailed visualization of GI tract structure and intestinal tumor nodules [78].

\subsection{DNA and related synthetic mimics}

DNA and artificial DNA-like molecules, e.g., peptide nucleic acid (PNA), locked nucleic acid (LNA), and phosphoramidate morpholino oligomers (MORF), have been investigated over the last several years as a class of powerful templates for synthesizing metal NCS with improved photophysical and biomedical performance [79-85]. The possibility to exploit several advantages, including tailored sequences and structures, tuneable size, and the presence of multiple coordination sites for metal ions, made DNA strands very appealing for the design of a wide variety of hybrid NC-based systems, with a proper modulation of their luminescence properties. For example, DNA-stabilized silver nanoclusters (DNA-Ag NCs) showed promising emission properties that spanned from the visible to the NIR region, depending on the type of DNA strand used [86, 87].

DNA-templated NCs have been widely studied as probes for the detection of specific biomarkers involved in complex biological events and diseases. Mucin-1 (MUC1) is a transmembrane glycoprotein with extensive O-linked glycosylation of its extracellular domain. MUC1 plays a central role in cellular protection, and is physiologically present on the surface of epithelial cells of lung, stomach, intestines, eyes, and a variety of hematopoietic cells. Since overexpression of MUC1 is commonly observed in many malignant neoplasms from breast, pancreatic, lung, prostate, and ovarian cancer, with fluorescein-labeled CTPR-Cu NCs $(\lambda \operatorname{exc}=405 \mathrm{~nm})$. Reproduced with permission from ref. [76]

its monitoring is extremely meaningful for early diagnosis and cancer treatment. Based on these premises, fluorescent DNA-templated Ag NCs was synthesized starting from a long single-stranded DNA core sequence that contained also a specifically designed MUC1-recognizing sequence. While the core sequence acted as template to control the synthesis of metal nanoclusters, the G-rich recognition sequence significantly improved their fluorescence intensity. This effect was removed upon MUC1 binding, inducing a clear fluorescence decrease and an outstanding limit of detection of $0.05 \mathrm{nM}$, which was successfully employed for monitoring MUC1 in serum and for imaging of MCF-7 breast cancer cells [88]. The abnormal expression of specific MicroRNA (miRNA) in biological fluids is another factor closely related to the occurrence of a variety of human diseases, such as malignant tumors and cardiovascular and neurological disease. Therefore, ultra-sensitive and selective detection of specific miRNAs is extremely relevant for early diagnostic screening $[89,90]$. In this context, $\mathrm{Li}$ et al. succeeded in developing DNA-Ag NCs able to detect traumatic brain injury (TBI) biomarkers. In particular, the Authors focused on Let-7i, a kind of TBI-related miRNA that significantly increases in the serum after injury events. To improve efficiency and sensitivity of their NC probe, a self-assembled DNA hairpin template-consisting of a cytosine-rich oligonucleotide, a loop-sequence acting as recognition site for Let-7i, and a folding domain for the stabilization of DNA hairpin structure-was used for the synthesis of Ag NCs (Fig. 5). These multifunctional DNA-Ag NCs showed good water dispersibility, low toxicity, and good photostability, exhibiting red fluorescence in Hela cells. Moreover, in the presence of Let-7i, a DNA hybridization reaction took place, and induced a fluorescence quenching, allowing a rapid Let$7 \mathrm{i}$ detection and quantification in vitro [91]. Another step forward in the implementation of smart metal NC-based bioimaging assays has recently been achieved by Yu et al., that 


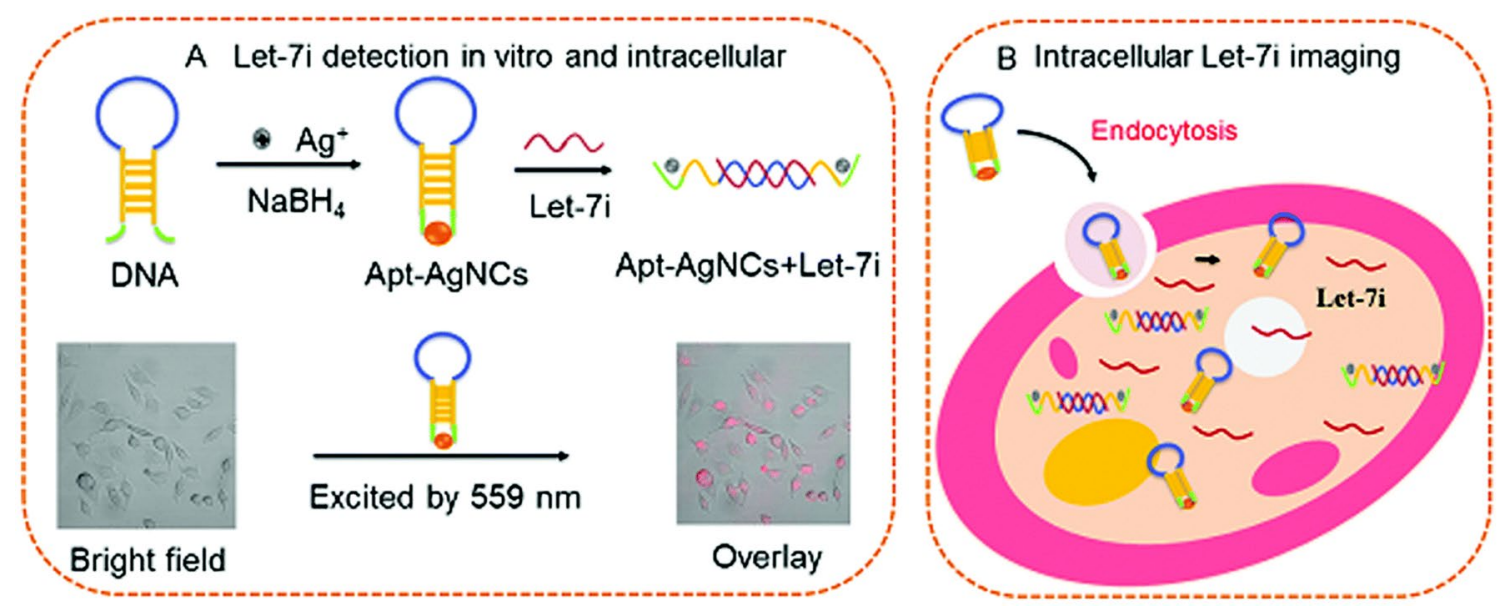

Fig. 5 Synthetic scheme of DNA hairpin silver nanoclusters (DNA$\mathrm{Ag} \mathrm{NCs)}$ as fluorescent probes for Let-7i detection, and related confocal fluorescence images obtained after internalization in $\mathrm{HeLa}$

developed the first Apt-DNA-Au nanoplatform able to detect mRNA-TK1 (TK1 = thymidine kinase 1 , a protein released in high amounts during tumor growth), and to allow realtime monitoring of breast cancer progression through the variation of NC fluorescence intensity [92].

\subsection{Biocompatible polymers}

Biocompatible polymers have emerged during the past decades, thanks to their excellent performance in a wide range of diagnostic and therapeutic medical devices. In addition to biodegradability and biocompatibility, they contain several functional groups that allow to control the assembly of metal NCs, and to optimize their structures, photophysical properties, and functionalities [33]. To improve the native fluorescence of Au NCs, Hembury et al. constrained the gold metal core within micelles formed by a thermosensitive copolymer of poly( $N$-isopropylacrylamide) (PNIPAm) and poly(ethylene glycol) (PEG). The gold-polymer nanohybrids were stable in biological environments and showed enhanced fluorescence at physiological conditions, with a temperature-dependent emission profile centered at $720 \mathrm{~nm}$. Fluorescence imaging proved that PEG-PNIPAm-Au NCs localized within vesicular structures inside HepG2 cells, paving the way to their potential use as fluorescent liveimaging contrast agents [93].

In the latest years, overuse of PEG-based polymers in health care has led to an increase in the release of anti-PEG antibodies and to the occurrence of severe allergic reactions in final users of such products. For these reasons, despite the great versatility and biocompatibility of PEG, there has been a growing demand for potential alternatives [94]. In this context, functionalized poly-(cyclic imino ether)s (PCIEs) have proven to be interesting candidates as PEG substitutes. cells (a). Proposed intracellular mechanism via cell endocytosis (b). Reproduced with permission from ref. [91]

Deepang and colleagues employed xanthene-PCIE derivatives to synthesize Au NCs, exploiting the thiol-like affinity of xanthene group toward metals and metal ions. The Authors demonstrated that an electrostatically stabilized core-shell interaction occurred between gold ions and the xanthate group, producing high-quality ultra-small PCIE$\mathrm{Au}$ NCs under mild conditions. PCIE-Au NCs showed stable fluorescence across a wide range of $\mathrm{pH}$ values and physiological conditions, with a narrow emission peak centered at $640 \mathrm{~nm}$. Moreover, in vivo experiments evidenced their cytocompatibility, low protein binding tendency, and reduced non-specific accumulation in liver and spleen [95].

A further approach to improve stability and bioavailability of metal nanoclusters is the use of naturally available biopolymers that protect the metal core from reaction with ROS and enzyme-containing intracellular milieu. For example, the use of chitosan as coating template was reported to improve resistance of $\mathrm{Au}$ NCs toward reactive oxygen species and proteases. Duan et al. designed a chitosan- $N$-acetyl-L-cysteine (NAC-CS) biocompatible polymer, and applied it to the synthesis of NAC-CScoated fluorescent gold nanoclusters (NAC-CS-Au NCs) with low cytotoxicity and long-time imaging responses in HeLa cells. Compared with their BSA-coated analogs, NAC-CS-Au NCs showed better stability toward hydrogen peroxide and trypsin. In vivo biodistribution tests highlighted that their NIR signal $\left(\lambda_{\max }=680 \mathrm{~nm}\right)$ was still detectable in the liver and kidney of normal mice after $6 \mathrm{~h}$ from injection [96]. A similar approach was used by Yang et al. in the template synthesis of bimetallic NCs, by grafting positive-charged CS with stearic acid (SA). The obtained amphiphilic fragment (ACD) self-assembled into micelles characterized by a hydrophobic pocket that allowed solubilization of $\mathrm{Pt}_{1} \mathrm{Ag}_{28} \mathrm{NCs}$ and enhanced $\mathrm{AIE}$ 


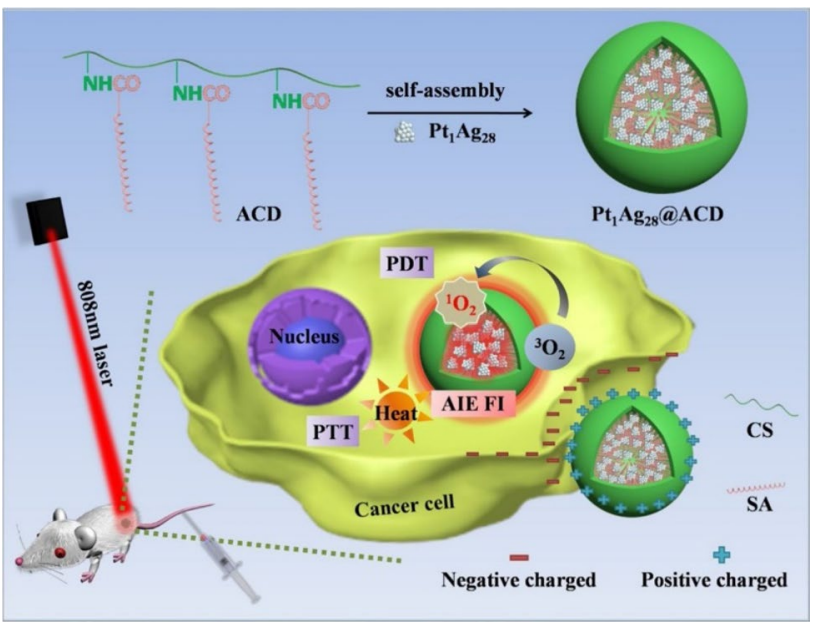

Fig. 6 Pictorial representation of self-assembled $\mathrm{Pt}_{1} \mathrm{Ag}_{28} @ \mathrm{ACD}$ structures, and their AIE effect in vivo. Reproduced with permission from ref. [97]

effect due to space restriction (Fig. 6). Photoluminescence spectra of $\mathrm{Pt}_{1} \mathrm{Ag}_{28} @ \mathrm{ACD}$ platforms exhibited an emission band, centered at $680 \mathrm{~nm}$, almost three times more intense than free $\mathrm{Pt}_{1} \mathrm{Ag}_{28}$. Interestingly, in vivo experiments on tumor-bearing mice showed selective accumulation of $\mathrm{Pt}_{1} \mathrm{Ag}_{28} @ \mathrm{ACD}$ micelles in tumor cells, and reduced nonspecific accumulation in heart and kidney, indicating that the NCs could passively target negatively charged cancers cells thanks to the presence of positive tails on micelle surface [97]. A different strategy to enhanced fluorescence stability toward $\mathrm{pH}$ changes and enzymatic degradation, consisted in the use of gelatin, a very valuable biopolymer for tissue engineering applications which is prepared by thermal denaturation of collagen. Using type B gelatin, Au NCs emitting at $640 \mathrm{~nm}$ were prepared and proved to have good compatibility toward human keratinocyte cell lines, allowing imaging and tracking in tissues by means of confocal laser scanning microscopy [98].

$\mathrm{Au}$ NCs have also been copolymerized with acrylamide and $\mathrm{N}$-acryloyl glycinamide polymers by a free radical polymerization reaction, in order to design a stimuliresponsive hydrogel with photo- and temperature-responsive properties. This composite hydrogel could be further integrated with hyaluronic acid as targeting molecule, obtaining an integrated platform for the capture and light-controlled release of MDA-MB-231 cancer cells [99]. Finally, an interesting example of methylcellulose (MC)-based optical fibers, containing Au@BSA and Au@GSH, for biological and medical applications has been reported by Nonappa et al. The presence of gold nanoclusters reinforced such solid fibers, and matched the surrounding tissue's dynamic mechanical properties with a suitable NC characteristic photoluminescence [100].

\section{Conclusions and perspectives}

Atomically precise metal nanoclusters have recently emerged as a novel class of luminescent nanomaterials with promising potential for biomedical applications, thanks to their ultra-small $(<2 \mathrm{~nm})$ size, excellent photostability, and good biocompatibility. The outstanding optical properties of NCs offer attractive chances for the design of optical sensors, photosensitizers, and light-emitting devices, but also for biolabeling and bioimaging purposes. In particular, the possibility to tune their PL all over the visible and NIR region by controlling the core size and the nature of stabilizing ligands makes NCs suitable candidates for deep tissue imaging and theranostic applications.

Among all the classes of templating ligands that has been tested in the preparation of tailored metal NCs, many research efforts has lately focused on the use of compounds of biological origin, eventually after proper chemical modification, including peptides, proteins, nucleic acids, and other biocompatible polymers. Apart from the stabilization and size tuning of metal cluster cores, the presence of such derivatives on the surface of NCs has been demonstrated to improve their biocompatibility, as well as to provide metal NCs with additional functionalities. For instance, the resulting hybrid NC-based platforms can exhibit specific targeting ability toward certain types of biomarkers, stimuli-responsive emission properties, or enhanced selectivity in localizing some particular cell substructure, tissue, or organ.

However, there are still some major challenges hampering the practical use of NCs in the biomedical field that need to be addressed. First of all, emission quantum yields of metal NCs are often not competitive, if compared to organic dyes and semiconductor quantum dots, and should be improved. Another issue requiring deeper studies is the currently still limited knowledge of the interactions between metal NCs and biomacromolecules, such as proteins, which is a fundamental step for fully understanding their biodistribution paths and in vivo potential toxicity.

Acknowledgements The authors are thankful to the NEWMED project, ID: 1175999 (funded by Regione Lombardia POR FESR 2014 2020) and to the NiFTy project funded by MIUR (PRIN2017, no. 2017MYBTXC).

\section{Declarations}

Conflict of interest The authors declare no conflict of interest.

\section{References}

1. Won, M., Li, M., Kim, H. S., Liu, P., Koo, S., Son, S., Seo, J. H., \& Kim, J. S. (2021). Visible to mid IR: a library of multispectral 
diagnostic imaging. Coordination Chemistry Reviews, 426, 213608. https://doi.org/10.1016/j.ccr.2020.213608

2. Colas, K., Doloczki, S., Posada Urrutia, M., \& Dyrager, C. (2021). Prevalent bioimaging scaffolds: synthesis, photophysical properties and applications. European Journal of Organic Chemistry, 15, 2133-2144. https://doi.org/10.1002/ejoc.20200 1658

3. Cardarelli, F. (2020). Back to the future: Genetically encoded fluorescent proteins as inert tracers of the intracellular environment. International Journal of Molecular Sciences, 21, 4164-4180. https://doi.org/10.3390/ijms21114164

4. Deng, H., Yan, S., Huang, Y., Lei, C., \& Nie, Z. (2020). Design strategies for fluorescent proteins/mimics and their applications in biosensing and bioimaging. Trends in Analytical Chemistry, 122, 115757. https://doi.org/10.1016/j.trac.2019.115757

5. Biswas, M. C., Islam, M. T., Nandy, P. K., \& Hossain, M. M. (2021). Graphene quantum dots (GQDs) for bioimaging and drug delivery applications: a review. ACS Materials Letters, 3, 889-911. https://doi.org/10.1021/acsmaterialslett.0c00550

6. Chung, S., Revia, R. A., \& Zhang, M. (2021). Graphene quantum dots and their applications in bioimaging, biosensing, and therapy. Advanced Materials, 33, 1904362. https://doi.org/10. 1002/adma.201904362

7. Ali, H., Ghosh, S., \& Jana, N. R. (2020). Fluorescent carbon dots as intracellular imaging probes. WIREs Nanomedicine \&amp; Nanobiotechnology, 12(e1617), 1-15. https://doi.org/10.1002/ wnan. 1617

8. Wan, J., Zhang, X., Zhang, K., \& Su, Z. (2020). Biological nanoscale fluorescent probes: From structure and performance to bioimaging. Reviews in Analytical Chemistry, 39, 209-221. https://doi.org/10.1515/revac-2020-0119

9. Peveler, W. J., Xiao, Z., Tsai, H.-Y., Gupta, R., Lix, K., Tran, M. V., \& Kim, H. (2021). Photoluminescent nanoparticles for chemical and biological analysis and imaging. Chemical Reviews, 121, 9243-9358. https://doi.org/10.1021/acs.chemrev.0c01176

10. Si, P., Razmi, N., Nur, O., Solanki, S., Pandey, C. M., Gupta, R. K., Malhotra, B. D., Willander, M., \& de la Zerda, A. (2021). Gold nanomaterials for optical biosensing and Bioimaging. Nanoscale Advances, 3, 2679-2698.

11. Jin, R., Zeng, C., Zhou, M., Chen, Y. (2016). Atomically precise colloidal metal nanoclusters and nanoparticles: Fundamentals and opportunities. Chemical Reviews, 116, 10346-10413. https:// doi.org/10.1021/acs.chemrev.5b00703

12. Yin, B., \& Luo, Z. (2021). Coinage metal clusters: From superatom chemistry to genetic materials. Coordination Chemistry Reviews, 429, 213643. https://doi.org/10.1016/j.ccr.2020.213643

13. Su, Y., Xue, T., Liu, Y., Qi, J., Jin, R., \& Lin, Z. (2019). Luminescent metal nanoclusters for biomedical applications. Nano Research, 12, 1251-1265. https://doi.org/10.1007/ s12274-019-2314-y

14. Zhang, L., \& Wang, E. (2014). Metal nanoclusters: New fluorescent probes for sensors and bioimaging. Nano Today, 9, 132-157. https://doi.org/10.1016/j.nantod.2014.02.010

15. Jin, R. (2015). Atomically precise metal nanoclusters: stable sizes and optical properties. Nanoscale, 7, 1549-1565. https://doi.org/ 10.1039/C4NR05794E

16. Zhao, Y., Zhou, H., Zhang, S., \& Xu, J. (2020). The synthesis of metal nanoclusters and their applications in bio-sensing and imaging. Methods and Applications in Fluorescence, 8, 012001. https://doi.org/10.1088/2050-6120/ab57e7

17. Higaki, T., Li, Q., Zhou, M., Zhao, S., Li, Y., Li, S., \& Jin, R. (2018). Toward the tailoring chemistry of metal nanoclusters for enhancing functionalities. Accounts of Chemical Research, 51, 2764-2773. https://doi.org/10.1021/acs.accounts.8b00383

18. Maity, S., Bain, D., \& Patra, A. (2019). An overview on the current understanding of the photophysical properties of metal nanoclusters and their potential applications. Nanoscale, 11, 22685-22723. https://doi.org/10.1039/C9NR07963G

19. Yang, T.-Q., Peng, B., Shan, B.-Q., Zong, Y.-X., Jiang, J.-G., Wu, P., \& Zhang, K. (2020). Origin of the photoluminescence of metal nanoclusters: from metal-centered emission to ligandcentered emission. Nanomaterials, 10, 261. https://doi.org/10. 3390/nano10020261

20. Díaz, S. A., Hastman, D. A., Medintz, I. L., \& Oh, E. (2017). Understanding energy transfer with luminescent gold nanoclusters: a promising new transduction modality for biorelated applications. Journal of Materials Chemistry B, 5, 7907-7926. https:// doi.org/10.1039/C7TB01654A

21. Crawford, S. E., Hartmann, M. J., \& Millstone, J. E. (2019). Surface chemistry-mediated near-infrared emission of small coinage metal nanoparticles. Accounts of Chemical Research, 52, 695-703. https://doi.org/10.1021/acs.accounts.8b00573

22. Goswami, N., Yao, Q., Luo, Z., Li, J., Chen, T., \& Xie, J. (2016). Luminescent metal nanoclusters with aggregation-induced emission. Journal of Physical Chemistry Letters, 7, 962-975. https:// doi.org/10.1021/acs.jpclett.5b02765

23. Yau, S. H., Varnavski, O., \& Goodson, T., III. (2013). An ultrafast look at Au nanoclusters. Accounts of Chemical Research, 46(7), 1506-1516. https://doi.org/10.1021/ar300280w

24. Yau, S. H., Abeyasinghe, N., Orr, M., Upton, L., Varnavski, O., Werner, J. H., Yeh, H.-C., Sharma, J., Shreve, A. P., Martinez, J. S., \& Goodson, T., III. (2012). Bright two-photon emission and ultra-fast relaxation dynamics in a DNA-templated nanocluster investigated by ultra-fast spectroscopy. Nanoscale, 4, 4247-4254. https://doi.org/10.1039/C2NR30628J

25. Ramakrishna, G., Varnavski, O., Kim, J., Lee, D., \& Goodson, T. (2008). Quantum-sized gold clusters as efficient two-photon absorbers. Journal of the American Chemical Society, 130, 5032-5033. https://doi.org/10.1021/ja800341v

26. Russier-Antoine, I., Bertorelle, F., Vojkovic, M., Rayane, D., Salmon, E., Jonin, C., Dugourd, P., Antoine, R., \& Brevet, P.-F. (2014). Non-linear optical properties of gold quantum clusters. The smaller the better. Nanoscale, 6, 13572-13578. https://doi. org/10.1039/C4NR03782K

27. Yousefalizadeh, G., Ahmadi, S., Mosey, N. J., \& Stamplecoskie, K. G. (2021). Exciting clusters, what does off-resonance actually mean? Nanoscale, 13, 242-252. https://doi.org/10.1039/D0NR0 6493A

28. Ho-Wu, R., Yau, S. H., \& Goodson, T., III. (2016). Linear and nonlinear optical properties of monolayer-protected gold nanocluster films. ACS Nano, 10, 562-572. https://doi.org/10.1021/ acsnano.5b05591

29. Russier-Antoine, I., Bertorelle, F., Calin, N., Sanader, Z., Krstić, M., Comby-Zerbino, C., Dugourd, P., Brevet, P.-F., BonačićKoutecký, V., \& Antoine, R. (2017). Ligand-core NLO-phores: a combined experimental and theoretical approach to the twophoton absorption and two-photon excited emission properties of small-ligated silver nanoclusters. Nanoscale, 9, 1221-1228. https://doi.org/10.1039/C6NR07989J

30. Chai, O. J. H., Liu, Z., Chen, T., \& Xie, J. (2019). Engineering ultrasmall metal nanoclusters for photocatalytic and electrocatalytic applications. Nanoscale, 11, 20437-20448. https://doi.org/ 10.1039/C9NR07272A

31. Du, X., \& Jin, R. (2019). Atomically precise metal nanoclusters for catalysis. ACS Nano, 13, 7383-7387. https://doi.org/10.1021/ acsnano.9b04533

32. Kwak, K., \& Lee, D. (2019). Electrochemistry of atomically precise metal nanoclusters. Accounts of Chemical Research, 52, 12-22. https://doi.org/10.1021/acs.accounts.8b00379

33. Rival, J. V., Mymoona, P., Lakshmi, K. M., Nonappa, Pradeep, T., \& Shibu, E. S. (2021). Self-assembly of precision noble metal nanoclusters: Hierarchical structural complexity, colloidal 
superstructures, and applications. Small, 2005718. https://doi. org/10.1002/smll.202005718

34. Dichiarante, V., Pigliacelli, C., Metrangolo, P., \& Baldelli Bombelli, F. (2021). Confined space design by nanoparticle selfassembly. Chemical Science, 12, 1632-1646. https://doi.org/10. 1039/D0SC05697A

35. Shellaiah, M., \& Sun, K. W. (2017). Luminescent metal nanoclusters for potential chemosensor applications. Chemosensors, 5, 36. https://doi.org/10.3390/chemosensors5040036

36. Lin, Z., Goswami, N., Xue, T., Chai, O. J. H., Xu, H., Liu, Y., Su, Y., \& Xie, J. (2021). Engineering metal nanoclusters for targeted therapeutics: from targeting strategies to therapeutic applications. Advanced Functional Materials, 31, 2105662. https://doi.org/10. 1002/adfm.202105662

37. Zheng, K., \& Xie, J. (2020). Engineering ultrasmall metal nanoclusters as promising theranostic agents. Trends in Chemistry, 2, 665-679. https://doi.org/10.1016/j.trechm.2020.04.011

38. Adabi, M., Naghibzadeh, M., Adabi, M., Zarrinfard, M. A., Esnaashari, S. S., Seifalian, A. M., Faridi-Majidi, R., Aiyelabegan, H. T., \& Ghanbari, H. (2017). Biocompatibility and nanostructured materials: applications in nanomedicine. Artificial Cells, Nanomedicine, and Biotechnology, 45, 833-842. https:// doi.org/10.1080/21691401.2016.1178134

39. Sengul, A. B., \& Asmatulu, E. (2020). Toxicity of metal and metal oxide nanoparticles: a review. Environmental Chemistry Letters, 18, 1659-1683. https://doi.org/10.1007/ s10311-020-01033-6

40. Zhang, X.-D., Wu, D., Shen, X., Liu, P.-X., Fan, F.-Y., \& Fan, S.-J. (2012). In vivo renal clearance, biodistribution, toxicity of gold nanoclusters. Biomaterials, 33, 4628-4638. https://doi.org/ 10.1016/j.biomaterials.2012.03.020

41. Wang, J.-Y., Chen, J., Yang, J., Wang, H., Shen, X., Sun, Y.-M., Guo, M., \& Zhang, X.-D. (2016). Effects of surface charges of gold nanoclusters on long-term in vivo biodistribution, toxicity, and cancer radiation therapy. International Journal of Nanomedicine, 11, 3475-3485. https://doi.org/10.2147/IJN.S106073

42. Loynachan, C. N., Soleimany, A. P., Dudani, J. S., Lin, Y., Najer, A., Bekdemir, A., Chen, Q., Bhatia, S. N., \& Stevens, M. M. (2019). Renal clearable catalytic gold nanoclusters for in vivo disease monitoring. Nature Nanotechnology, 14, 883-890. https://doi.org/10.1038/s41565-019-0527-6

43. Qiao, Z., Zhang, J., Hai, X., Yan, Y., Song, W., \& Bi, S. (2021). Recent advances in templated synthesis of metal nanoclusters and their applications in biosensing, bioimaging and theranostics. Biosensors and Bioelectronics, 176, 112898. https://doi.org/10. 1016/j.bios.2020.112898

44. Yang, W., Guo, W., Chang, J., \& Zhang, B. (2017). Protein/peptide-templated biomimetic synthesis of inorganic nanoparticles for biomedical applications. Journal of Materials Chemistry B, 5, 401-417. https://doi.org/10.1039/C6TB02308H

45. Kailasa, S. K., Borse, S., Koduru, J. R., \& Murthy, Z. V. P. (2021). Biomolecules as promising ligands in the synthesis of metal nanoclusters: sensing, bioimaging and catalytic applications. Trends in Environmental Analytical Chemistry, 32, e00140. https://doi.org/10.1016/j.teac.2021.e00140

46. Romeo, V., Lopez-Martınez, E., Berganza-Granda, J., Gonide-Cerio, F., \& Cortajarena, A. L. (2021). Biomarker sensing platforms based on fluorescent metal nanoclusters. Nanoscale Advances, 3, 1331-1341. https://doi.org/10.1039/D0NA00796J

47. Wang, B., Zhao, M., Mehdi, M., Wang, G., Gao, P., \& Zhang, K. Q. (2019). Biomolecule-assisted synthesis and functionality of metal nanoclusters for biological sensing: a review. Materials Chemistry Frontiers, 3, 1722-1732. https://doi.org/10.1039/ C9QM00165D

48. Kundu, S., Mukherjee, D., Maiti, T. K., \& Sarkar, N. (2019). Highly luminescent thermoresponsive green emitting gold nanoclusters for intracellular nanothermometry and cellular imaging: a dual function optical probe. ACS Applied Bio Materials, 2, 2078-2091. https://doi.org/10.1021/acsabm.9b00107

49. Oliver-Cervelloo, L., Martin-Gomez, H. \& Mas-Moruno, C. (2021). New trends in the development of multifunctional peptides to functionalize biomaterials, Journal of Peptide Science, e3335. https://doi.org/10.1002/psc.3335

50. Zhao, J., \& Yang, P. (2020). Amyloid-mediated fabrication of organic-inorganic hybrid materials and their biomedical applications. Advanced Materials Interfaces, 7, 2001060. https:// doi.org/10.1002/admi.202001060

51. Ke, P. C., Sani, M. A., Ding, F., Kakinen, A., Javed, I., Separovic, F., Davis, T. P., \& Mezzenga, R. (2017). Implications of peptide assemblies in amyloid diseases. Chemical Society Reviews, 46, 6492-6531. https://doi.org/10.1039/C7CS00372B

52. Zhang, S., Zhang, X., \& Su, Z. (2020). Biomolecule conjugated metal nanoclusters: bio-inspiration strategies, targeted therapeutics, and diagnostics. Journal of Materials Chemistry B, 8 , 4176-4194. https://doi.org/10.1039/C9TB02936B

53. Wang, Y., Xia, K., Wang, L., Wu, M., Sang, X., Wan, K., Zhang, X., Liu, X., \& Wei, G. (2021). Peptide-engineered fluorescent nanomaterials: structure design, function tailoring, and biomedical applications. Small (Weinheim an der Bergstrasse, Germany), 17, 2005578. https://doi.org/10.1002/smll.20200 5578

54. Tarvirdipour, S., Huang, X., Mihali, V., Schoenenberger, C. A., \& Palivan, C. G. (2020). Peptide-based nanoassemblies in gene therapy and diagnosis: paving the way for clinical application. Molecules, 25, 3482. https://doi.org/10.3390/molecules251534 82

55. Yao, Q., Wu, Z., Liu, Z., Lin, Y., Yuan, X., \& Xie, J. (2021). Molecular reactivity of thiolate-protected noble metal nanoclusters: synthesis, self-assembly, and applications. Chemical Science, 12, 99-127. https://doi.org/10.1039/D0SC04620E

56. Das, N. M., Ghosh, S., Priya, A., Datta, S., \& Mukherjee, S. (2015). Luminescent copper nanoclusters as a specific cell-imaging probe and a selective metal ion sensor. Journal of Physical Chemistry C, 119, 24657-24664. https://doi.org/10.1021/acs. jpcc.5b08123

57. Zhang, H., Han, W., Cao, X., Gao, T., Jia, R., Liu, M., \& Zeng, W. (2019). Gold nanoclusters as a near-infrared fluorometric nanothermometer for living cells. Microchimica Acta, 186, 353. https://doi.org/10.1007/s00604-019-3460-3

58. Kundu, S., Ghosh, B., Nandi, S., Ghosh, M., Pyne, A., Chatterjee, J., \& Sarkar, N. (2020). Surface ligand-controlled wavelengthtunable luminescence of gold nanoclusters: cellular imaging and smart fluorescent probes for amyloid detection. ACS Applied Bio Materials, 3, 4282-4293. https://doi.org/10.1021/acsabm.0c003 37

59. You, J. G. \& Tseng, W. L. (2019). Peptide-induced aggregation of glutathione-capped gold nanoclusters: A new strategy for designing aggregation-induced enhanced emission probes. Analytica Chimica Acta, 1078, 101e111. https://doi.org/10.1016/j.aca. 2019.05.069.

60. Broekgaarden, M., Bulin, A. L., Porret, E., Musnier, B., Chovelon, B., Ravelet, C., Sancey, L., Elleaume, H., Hainaut, P., Coll, J. L., \& Le Guével, X. (2020). Surface functionalization of gold nanoclusters with arginine: a trade-off between microtumor uptake and radiotherapy enhancement. Nanoscale, 12 , 6959-6963. https://doi.org/10.1039/D0NR01138J

61. Wang, Y., Cui, Y., Zhao, Y., Liu, R., Sun, Z., Li, W., \& Gao, X. (2012). Bifunctional peptides that precisely biomineralize $\mathrm{Au}$ clusters and specifically stain cell nuclei. Chemical Communications, 48, 871-873. https://doi.org/10.1039/C1CC15926G

62. Yuan, Q., Wang, Y., Zhao, L., Liu, R., Gao, F., Gao, L., \& Gao, X. (2016). Peptide protected gold clusters: chemical synthesis 
and biomedical applications. Nanoscale, 8, 12095-12104. https:// doi.org/10.1039/C6NR02750D

63. Jia, M., Mi, W., Guo, S., Yang, Q. Z., Jin, Y., \& Shao, N. (2020). Peptide-capped functionalized $\mathrm{Ag} / \mathrm{Au}$ bimetal nanoclusters with enhanced red fluorescence for lysosome-targeted imaging of hypochlorite in living cells. Talanta, 216, 120926. https://doi. org/10.1016/j.talanta.2020.120926

64. Liu, C., Zhang, X., Han, X., Fang, Y., Liu, X., Wang, X., Waterhouse, G. I. N., Xu, C., Yin, C., \& Gao, X. (2020). Polypeptidetemplated Au nanoclusters with red and blue fluorescence emissions for multimodal imaging of cell nuclei. ACS Applied Bio Materials, 3, 1934-1943. https://doi.org/10.1021/acsabm.9b010 78

65. Zhang, W., Lin, D., Wang, H., Li, J., Nienhaus, J. U., Su, Z., Wei, G., \& Shang L. (2017). Supramolecular self-assembly bioinspired synthesis of luminescent gold nanocluster-embedded peptide nanofibers for temperature sensing and cellular imaging. Bioconjugate Chemistry, 28, 2224-2229. https://doi.org/10. 1021/acs.bioconjchem.7b00312

66. Chen, D., Li, D., Cai, S., Wang, P., Peng, S., Sheng, Y., He, Y., Gu, Y., \& Chen, H. (2016). Dual targeting luminescent gold nanoclusters for tumor imaging and deep tissue therapy. Biomaterials, 100, 1-16. https://doi.org/10.1016/j.biomaterials.2016. 05.017

67. Zare, I., Chevrier, D. M., Cifuentes-Rius, A., Moradi, N., Xianyu, Y., Ghosh, S., Trapiella-Alfonso, L., Tian, Y., ShourangizHaghighi, A., Mukherjee, S., Fan, K., \& Hamblin, M. R. (2021). Protein-protected metal nanoclusters as diagnostic and therapeutic platforms for biomedical applications. Materials Today, Advanced Online Publication. https://doi.org/10.1016/j.mattod. 2020.10.027

68. El-Sayed, N., \& Schneider, M. (2020). Advances in biomedical and pharmaceutical applications of protein-stabilized gold nanoclusters. Journal of Materials Chemistry B, 8, 8952-8971. https://doi.org/10.1039/D0TB01610A

69. Di Giosia, M., Zerbetto, F., \& Calvaresi, M. (2021). Incorporation of molecular nanoparticles inside proteins: The trojan horse approach in theranostics. Accounts of Materials Research, 2, 594-605. https://doi.org/10.1021/accountsmr.1c00065

70. Balu, R., Bourgeois, L., Elvin, C. M., Hill, A. J., Choudhurya, N. R., \& Dutta, N. K. (2015). A multi-responsive intrinsically disordered protein (IDP)-directed green synthesis of fluorescent gold nanoclusters. Journal of Materials Chemistry B, 3, 6580-6586. https://doi.org/10.1039/C5TB00659G

71. Xie, J., Zheng, Y., \& Ying, J. Y. (2009). Protein-directed synthesis of highly fluorescent gold nanoclusters. Journal of the American Chemical Society, 131, 888-889. https://doi.org/10. $1021 / \mathrm{ja} 806804 \mathrm{u}$

72. Jain, V., Bhagat, S., \& Singh, S. (2021). Bovine serum albumin decorated gold nanoclusters: A fluorescence-based nanoprobe for detection of intracellular hydrogen peroxide. Sensors \&amp; Actuators, 327, 128886. https://doi.org/10.1016/j.snb.2020. 128886

73. Wu, Y. T., Shanmugam, C., Tseng, W. B., Hiseh, M. M., \& Tseng, W. L. (2016). A gold nanocluster-based fluorescent probe for simultaneous $\mathrm{pH}$ and temperature sensing and its application to cellular imaging and logic gates. Nanoscale, 8,11210 . https:// doi.org/10.1039/C6NR02341J

74. Cheung, A., Bax, H. J., Josephs, D. H., Ilieva, K. M., Pellizzari, G., Opzoomer, J., Bloomfield, J., Fittall, M., Grigoriadis, A., Figini, M., Canevari, S., Spicer, J. F., Tutt, A. N. \& Karagiannis, S. N. (2016). Targeting folate receptor alpha for cancer treatment. Oncotarget, 7, 52553-52574. https://doi.org/10.18632/ oncotarget.9651

75. Hada, A. M., Craciun, A. N., Focsan, M., Borlan, R., Soritau, O., Todea, M., \& Astilean, S. (2021). Folic acid functionalized gold nanoclusters for enabling targeted fluorescence imaging of human ovarian cancer cells. Talanta, 225, 121960. https:// doi.org/10.1016/j.talanta.2020.121960

76. Aires, A., Llarena, I., Moller, M., Castro-Smirnov, J., Cabanillas-Gonzalez, J., \& Cortajarena, A. L. (2019). A simple approach to design proteins for the sustainable synthesis of metal nanoclusters. Angewandte Chemie International Edition, 58, 6214-6219. https://doi.org/10.1002/anie.201813576

77. Aires, A., Maestro, D., Ruiz del Rio, J., Palanca, A. R., LopezMartinez, E., Llarena, I., Geraki, K., Sanchez- Cano, C., Villar, A. V., \& Cortajarena, A. L. (2021). Engineering multifunctional metal/protein hybrid nanomaterials as tools for therapeutic intervention and high-sensitivity detection. Chemical Science, 12, 2480-2487. https://doi.org/10.1039/D0SC05215A

78. Wang, W., Kong, Y., Jiang, J., Xie, Q., Huang, Y., Li, G., Wu, D., Zheng, H., Gao, M., Xu, S., Pan, Y., Li, W., Ma, R., Wu, M. X., Li, X., Zuilhof, H., Cai, X., \& Li, R. (2020). Engineering the protein corona structure on gold nanoclusters enables red-shifted emissions in the second near-infrared window for gastrointestinal imaging. Angewandte Chemie International Edition, 59, 22431-22435. https://doi.org/10.1002/anie.20201 0089

79. Chen, Y., Phipps, M. L., Werner, J. H., Chakraborty, S. \& Martinez, J. S., DNA templated metal nanoclusters: From emergent properties to unique applications. Accounts of Chemical Research, 51, 2756-2763. https://doi.org/10.1021/acs.accounts. $8 \mathrm{~b} 00366$

80. Guo, Y., Pan, X., Zhang, W., Hu, Z., Wong, K. W., He, Z., \& $\mathrm{Li}, \mathrm{H}$. W. (2020). Label-free probes using DNA-templated silver nanoclusters as versatile reporters. Biosensors and Bioelectronics, 150, 111926. https://doi.org/10.1016/j.bios.2019.111926

81. Pandya, A., Lad, A. N., Singh, S. P., \& Shanker, R. (2016). DNA assembled metal nanoclusters: synthesis to novel applications. RSC Advances, 6, 113095-113114. https://doi.org/10.1039/ C6RA24098D

82. Qing, Z., Bai, A., Xing, S., Zou, Z., He, X., Wang, K., \& Yang, R. (2019). Progress in biosensor based on DNA-templated copper nanoparticles. Biosensors and Bioelectronics, 137, 96-109. https://doi.org/10.1016/j.bios.2019.05.014

83. Geczy, R., Christensen, N. J., Rasmussen, K. K., Kalomista, I., Tiwari, M. K., Shah, P., Yang, S. W., Bjerrum, M. J., \& Thulstrup, P. W. (2020). Formation and structure of fluorescent silver nanoclusters at interfacial binding sites facilitating oligomerization of DNA hairpins. Angewandte Chemie International Edition, 59, 16091-16097. https://doi.org/10.1002/anie.202005102

84. Xu, F., Qing, T., \& Qing, Z. (2021). DNA-coded metal nanofluorophores: preparation, properties and applications in biosensing and bioimaging. NanoToday, 36, 101021. https://doi.org/10. 1016/j.nantod.2020.101021

85. Wang, Y., Wang, X., Ma, X., Chen, Q., He, H., Nau, W. M., et al. (2019). Coassembly of gold nanoclusters with nucleic acids: sensing, bioimaging, and gene transfection. Particle \&amp; Particle Systems Characterization, 36, 1900281. https://doi.org/ 10.1002/ppsc.201900281

86. Yang, M., Chen, X., Su, Y., Liu, H., Zhang, H., Li, X., \& Xu, W. (2020). The fluorescent palette of DNA-templated silver nanoclusters for biological applications. Frontiers in Chemistry, 8, 601621. https://doi.org/10.3389/fchem.2020.601621

87. Cerretani, C., Kanazawa, H., Vosch, T., \& Kondo, J. (2019). Crystal structure of a NIR-emitting DNA-stabilized $\mathrm{Ag}_{16}$ nanocluster. Angewandte Chemie International Edition, 58, 1715317157. https://doi.org/10.1002/anie.201906766

88. Zhoua, L., Wang, W., Chen, Y., Fan, J., Tong, C., \& Liu, B. (2019). Aptamer-tagged silver nanoclusters for cell image and Mucin1 detection in vitro. Talanta, 205, 120075. https://doi.org/ 10.1016/j.talanta.2019.06.075 
89. Fang, B., Guo, P., Yang, M., Ma, Y., Yan, X., Jia, Z., Gao, W., Ahmad, S., Xu, C., Liu, C., \& Zhang, M. (2020). A novel fluorescent enhancing platform based on DNA-scaffolded silver nanoclusters for potential inflammatory bowel disease-associated microRNA detection. Talanta, 218, 121122. https://doi.org/10. 1016/j.talanta.2020.121122

90. Cai, Q., Li, H., Cao, W., \& Jie, G. (2021). Photoinduced-electron transfer coupled with DNA cross-chain displacement multiple amplification for fluorescence biosensing of MicroRNA. Analytica Chimica Acta, 1148, 238169. https://doi.org/10.1016/j.aca. 2020.12.044

91. Li, X., Han, L., Guo, Y., Chang, Y., Yan, J., Wang, Y., Li, N., Ding, Y., \& Cai, J. (2019). Rapid detection and cellular fluorescence imaging of the TBI biomarker Let-7i using a DNA-AgNC nanoprobe. New Journal of Chemistry, 43, 7997-8004. https:// doi.org/10.1039/C9NJ00489K

92. Yu, S., Zhou, Y., Sun, Y., Wu, S., Xu, T., Chang, Y. C., Bi, S., Jiang, L. P., \& Zhu, J. J. (2021). Endogenous mRNA triggered DNA-Au nanomachine for in situ imaging and targeted multimodal synergistic cancer therapy. Angewandte Chemie International Edition, 60, 5948-5958. https://doi.org/10.1002/anie. 202012801

93. Hembury, M., Beztsinna, N., Asadi, H., Van den Dikkenberg, J. B., Meeldijk, J. D., Hennink, W. E., \& Vermonden, T. (2018). Luminescent gold nanocluster-decorated polymeric hybrid particles with assembly-induced emission. Biomacromolecules, 19 , 2841-2848. https://doi.org/10.1021/acs.biomac.8b00414

94. Povsic, T. J., Lawrence, M. G., Lincoff, A. M., Mehran, R., Rusconi, C. P., Zelenkofske, S. L., Huang, Z., Sailstad, J., Armstrong, P. W., Steg, P. G., Bode, C., Becker, R. C., Alexander, J. H., Adkinson, N. F., \& Levinson, A. I. (2016). Pre-existing anti-PEG antibodies are associated with severe immediate allergic reactions to pegnivacogin, a PEGylated aptamer. Journal of Allergy and Clinical Immunology, 138, 1712-1715. https://doi. org/10.1016/j.jaci.2016.04.058
95. Deepagan, V. G., Leiske, M. N., Fletcher, N. L., Rudd, D., Tieu, T., Kirkwood, N., Thurecht, K. J., Kempe, K., Voelcker, N. H., \& Cifuentes-Rius, A. (2021). Engineering fluorescent gold nanoclusters using xanthate- functionalized hydrophilic polymers: toward enhanced monodispersity and stability. Nano Letters, 21, 476-484. https://doi.org/10.1021/acs.nanolett.0c03930

96. Duan, Y., Duan, R., Liu, R., Guan, M., Chen, W., Ma, J., Chen, M., Du, B., \& Zhang, Q. (2018). Chitosan-stabilized self-assembled fluorescent gold nanoclusters for cell imaging and biodistribution in vivo. ACS Biomaterials Science \&amp; Engineering, 4, 1055-1063. https://doi.org/10.1021/acsbiomaterials.7b00975

97. Yang, Y., Wang, S., Zhou, Y., Wang, X., Liu, X., Xie, A., Shen, Y., \& Zhu, M. (2020). Structurally accurate lipophilic Pt1Ag28 nanoclusters based cancer theranostic micelles for dual-targeting/ aggregation enhanced fluorescence imaging and photothermal/ photodynamic therapies. Colloids and Surfaces B: Biointerfaces, 196, 111346. https://doi.org/10.1016/j.colsurfb.2020.111346

98. El-Sayed, N., Trouillet, V., Clasen, A., Jung, G., Hollemeyer, K., \& Schneider, M. (2019). NIR-emitting gold nanoclustersmodified gelatin nanoparticles as a bioimaging agent in tissue. Advanced Healthcare Materials, 8, 1900993. https://doi.org/10. 1002/adhm.201900993

99. Zhu, S., Wang, X., Cong, Y., Liu, L., \& Li, L. (2021). Free radical polymerization of gold nanoclusters and hydrogels for cell capture and light-controlled release. ACS Applied Materials \&amp; Interfaces, 13, 19360-19368. https://doi.org/10.1021/acsami. $1 \mathrm{c} 03587$

100. Hynninen, V., Chandra, S., Das, S., Amini, M., Dai, Y., Lepikko, S., Mohammadi, P., Hietala, S., Ras, R. H. A., Sun, Z., Ikkala, O., \& Nonappa. (2021). Luminescent gold nanocluster-methylcellulose composite optical fibers with low attenuation coefficient and high photostability. Small (Weinheim an der Bergstrasse, Germany), 17, 2005205. https://doi.org/10.1002/smll.202005205

\section{Authors and Affiliations}

\section{Greta Bergamaschi ${ }^{1}$ (i) $\cdot$ Pierangelo Metrangolo ${ }^{2}$ (i) $\cdot$ Valentina Dichiarante ${ }^{2}$ (i)}

1 Istituto di Scienze e Tecnologie Chimiche "Giulio Natta", National Research Council of Italy (SCITEC-CNR), via M. Bianco 9, 20131 Milan, Italy

2 Laboratory of Supramolecular and Bio-Nanomaterials (SupraBioNanoLab), Department of Chemistry, Materials and Chemical Engineering "Giulio Natta", Politecnico di Milano, via L. Mancinelli 7, 20131 Milan, Italy 